\title{
À nouvelles technologies, nouvelle pédagogie
}

\author{
Jean-Louis Trouillon
}

\section{OpenEdition \\ Journals}

Édition électronique

URL : http://journals.openedition.org/asp/4345

DOI : 10.4000/asp.4345

ISSN : 2108-6354

\section{Éditeur}

Groupe d'étude et de recherche en anglais de spécialité

\section{Édition imprimée}

Date de publication : 1 octobre 1993

Pagination : 413-422

ISSN : 1246-8185

\section{Référence électronique}

Jean-Louis Trouillon, «À nouvelles technologies, nouvelle pédagogie », ASp [En ligne], 2 | 1993, mis en ligne le 07 mars 2014, consulté le 01 mai 2019. URL : http://journals.openedition.org/asp/4345 ; DOI : $10.4000 / a s p .4345$

Ce document a été généré automatiquement le 1 mai 2019.

Tous droits réservés 


\title{
À nouvelles technologies, nouvelle pédagogie
}

\author{
Jean-Louis Trouillon
}

1 J'emprunte le titre de cette communication à Jean-Michel Dusseau, physicien et spécialiste de nouvelles technologies et de didactique. Malgré la masse des expériences déjà effectuées, avec l'EAO en particulier, on entend en effet dire trop souvent encore que tel ou tel outil va, par un coup de baguette magique, permettre de résoudre comme par miracle ce que les enseignants n'ont jamais pu transmettre de façon satisfaisante.

2 Travaillant depuis neuf ans sur et avec des machines, aussi bien par goût que par nécessité, je pense qu'il est bon de rappeler une nouvelle fois qu'un outil pédagogique ne vaut que par son contenu didactique, par ceux qui l'utilisent et par l'utilisation que l'on en fait; l'outil pédagogique c'est un peu une automobile qui ne saurait répondre à sa vocation si elle n'a ni carburant approprié ni conducteur qualifié.

3 Je voudrais, au travers de deux exemples précis, essayer d'évoquer d'abord une méthodologie de critères de sélection d'outils en fonction des contraintes imposées mais aussi ensuite et surtout les implications didactiques qui découlent du choix ainsi réalisé. Mes deux exemples sont représentatifs non seulement de deux situations d'apprentissage différentes mais également de deux domaines différents : anglais du tourisme d'une part, lexique scientifique de base d'autre part. Tous deux furent réalisés en étroite collaboration avec le centre associé Languedoc Roussillon du Conservatoire National des Arts et Métiers.

\section{Le télécours d'anglais du tourisme}

\section{Le public}

4 Il s'agit d'un public de professionnels du tourisme, en grande majorité hôteliers et restaurateurs, situés dans une région très attirante et qui ne manque point donc d'attirer en été de nombreux visiteurs y compris des visiteurs étrangers. 
5 Toutefois, le département de la Lozère est, grâce à son climat et à sa géographie, une zone difficile. Dans un contexte de formation continue il est donc inenvisageable de demander à des professionnels de clore boutique, de parcourir jusqu'à $100 \mathrm{~km}$ (aller simple) deux ou trois fois par semaine pour se rendre dans un centre de regroupement. Le droit à la formation semble donc se heurter à des obstacles difficilement surmontables.

6 Notre rôle est donc d'abord, compte tenu des contraintes que je viens d'énoncer, de sélectionner les outils qui nous permettent d'assurer la formation demandée.

7 Fort heureusement, les nouvelles technologies sont là et offrent un choix vaste. Nous avons retenu le système dit du télécours.

\section{Le dispositif}

8 Ce système, développée par la société Genesys est une variante de la téléconférence. Reliés par le biais du RTC, Réseau Téléphonique Commuté c'est-à-dire le réseau habituel proposé par France TÉLÉCOM, à un pont téléphonique qui n'est autre qu'un serveur, les différents participants se retrouvent pour le cours à jour et heures fixes. Le rôle du pont est double : à un niveau technique tout d'abord il permet la gestion continue du télécours, par exemple les appels automatiques groupés ou individuels. Sur un plan pédagogique enfin, le pont autorise le téléchargement et la diffusion de scénarios c'est-à-dire, soit de séquences sonores pré-enregistrées soit de pages-écran lues par le Minitel des participants. Cette diffusion, commandée par les touches du combiné de l'animateur, est quasi instantanée et peut, à l'alternat, intervenir à tout moment du cours.

Pour un coût acceptable de formation à distance, ce système permet donc de pallier l'éloignement géographique.

\section{La pédagogie}

La question suivante est, nous avons le dispositif, qu'en faisons-nous? Pouvions-nous nous contenter de réutiliser au téléphone les techniques traditionnelles de salle de classe en ignorant ce qui pouvait différencier les situations? Évidemment non. La matière à enseigner, les objectifs, demeurent, mais les conditions changent du tout au tout, les relations entre élèves, entre enseignant et élèves aussi.

11 Une fois rappelés nos objectifs, le choix préliminaire de l'approche la plus appropriée ne pouvait en théorie s'opérer qu'en tenant compte des expériences passées. Mais l'utilisation du Minitel en direct parallèlement à un cours de langue de spécialité en temps réel était une première. Compte tenu de toute façon de la rareté des antécédents dans le domaine de l'enseignement d'une langue de spécialité par téléphone à un groupe et de la quasi-absence de publications sur ce sujet nous avons en fait surtout travaillé de façon imaginative et pragmatique, d'abord en réfléchissant à tout ce qui pouvait être une situation de communication authentique, immédiate et non visuelle.

Confortés en cela par les expériences poursuivies en d'autres matières, rappelées pour certaines d'entre elles en bibliographie, il nous a fallu concevoir ce que pouvait être une dynamique fondée uniquement sur la voix et sur le silence. Pour cela l'écoute de la radio a été un guide précieux. Si les longs monologues sont évidemment à exclure parce que lassants et totalement inadaptés à l'interactivité du moyen retenu, il n'est pas si évident de concevoir un ensemble d'apprentissage basé sur un échange continu: l'absence de 
repères visuels oblige à anticiper les réactions éventuelles de chacun. À cela il faut ajouter un besoin de discipline de tour de parole : tendance naturelle à l'effacement de la part de certains participants et au contraire apparition de «bavards» accaparant la parole. Les échanges eux-mêmes sont à adapter sur un système qui sans être aussi lourd et contraignant qu'une procédure radio aéronautique ou militaire ne doit pas laisser planer la moindre ambiguïté : on doit comprendre immédiatement qu'une question est une question ou qu'une réponse est terminée et entraîne une nouvelle prise de parole; l'intonation a donc fait l'objet de nombreux exercices prioritaires.

Il ne s'agit là que de quelques exemples qui montrent les voies vers lesquelles nous pouvons être amenés à nous engager lorsque nous pensons nouvelles technologies: les contraintes ne sont pas uniquement financières et institutionnelles, elles naissent également du support et régissent nos choix pédagogiques.

Quelques mots sur les résultats. Les objectifs initiaux, prudents il est vrai, ont été très largement dépassés. Une analyse des raisons du succès nous a donné les éléments suivants :

- motivation élevée car le besoin est réel et immédiat. Les participants ont tout particulièrement apprécié que nous ayons tenté d'apporter une solution adaptée à leurs besoins ;

- forte attention apportée à l'écoute car la moindre perte d'attention est immédiatement sanctionnée, on ne sait plus du tout où on en est. Cette attention soutenue a permis l'émergence immédiate d'un esprit de groupe, d'une situation très familiale et donc très libre, ce qui nous amène directement au point suivant ;

- paradoxalement pour nous, mais pas du tout pour les psychologues consultés, rôle désinhibiteur du téléphone. Les stagiaires étaient chez eux, dans un cadre familier et donc rassurant, hors de la situation salle de classe qui ne leur avait guère apporté le succès au cours de leurs études.

15 En conclusion de cette première partie, je voudrais dire que nous avons été tellement satisfaits du résultat, que ce qui avait commencé comme un simple objet de recherche devient une forme d'enseignement que nous développons à l'heure actuelle dans deux directions :

- reprise et continuation du télécours sous la forme que je viens d'évoquer avec des publics et des besoins différents ;

- la fonction laboratoire de langue : le combiné joue le rôle du casque, le cadran du téléphone émule les touches de commande d'un magnétophone. Le serveur consacre une partie de sa mémoire à l'enregistrement des séquences sonores des élèves afin que l'enseignant puisse, en temps différé, réécouter la production de ces derniers.

La conséquence pratique de cette extension est qu'une "vieille pédagogie ", adaptée à un support récent, peut connaître un renouveau. En effet, outre le simple transfert toujours possible d'exercices déjà réalisés pour un laboratoire traditionnel, ce télélabo doit aussi permettre la réalisation d'approches moins systématisées, moins techniques et plus conviviales: la spontanéité des échanges devrait en être accrue. Par une sorte de paradoxe, alors que j'ai dans le passé souvent considéré le laboratoire comme un palliatif du téléphone ce dernier va maintenant émuler le laboratoire. 


\section{EAO, auto-formation dirigée et lexique de spécialité}

17 Pourquoi un deuxième exemple ? Parce que je n'oublie pas le thème de notre rencontre et que je souhaite montrer que face à des contraintes différentes les choix seront, ce n'est pas original de le dire, très certainement différents, imposés peut-être.

18 Si les contraintes sont multiples, la liberté n'est en effet pas nécessairement infinie. Elle se heurte évidemment à des obstacles qui ne sont pas tous sonnants et trébuchants puisque l'expérience montre que lorsque l'on sait frapper aux bonnes portes pour proposer un projet cohérent en prise directe avec les besoins socio-économiques on trouve le financement nécessaire.

Beaucoup plus dures sont les contraintes du marché lorsqu'on souhaite non plus mettre sur pied une expérimentation, mais offrir un produit fini.

Je change donc de casquette, de public, de spécialité, de technique et vais rappeler quelques-uns des problèmes qui se posent lorsque l'objectif est un produit commercialisé.

\section{Objectifs}

21 Nous ne sommes plus en formation continue, mais à l'université, en 1987, et nous devons amener une formation de base en anglais scientifique au nombre sans cesse croissant d'étudiants qu'accueillent à bras ouverts nos universités sans que le Ministère accompagne cette ouverture d'une attribution des moyens en personnels et locaux que l'on serait en droit d'espérer. Seule solution apparente, le recours partiel à l'EAO.

\section{Moyens}

Pour ce faire il ne suffit pas d'ordinateurs, il faut aussi des didacticiels. Il n'existe pas sur le marché - nous sommes, je le rappelle, en 1987 - de didacticiels répondant à nos besoins. Nous décidons de créer ce dont nous avons besoin et nous mettons en quête de partenaires: d'abord pourvoyeurs de crédits, auteurs et techniciens, bientôt gestionnaires, et enfin éditeur et distributeur.

\section{L'élaboration de la solution}

23 Je passerai rapidement sur une contrainte qui nous fut imposée par l'un de nos bailleurs de fonds qui nous allouait une fort jolie somme à la seule condition que notre système fit appel à un réseau de type RNIS qu'il s'apprêtait à mettre en place. Nous développâmes donc pendant deux ans tout un ensemble conçu dans ce but. Malheureusement le réseau, après avoir coûté plusieurs fortunes aux contribuables, n'a jamais vu le jour. Nous nous trouvâmes donc non seulement fort dépourvus de support, mais en outre en butte à l'ire des autres partenaires qui avaient en partie financé le projet et ne voyaient rien venir.

Il fallait donc trouver une autre solution : fort heureusement, l'un des éditeurs pressentis, Mediaconcept, proposa de mettre à notre disposition les outils qu'il avait développés pour une de ses collections.

Le temps, autre contrainte majeure, passant, et ne lassant point nos commanditaires, nous avons alors choisi d'adapter notre production à la technique proposée. 
Le résultat est là, enfin : l'éditeur dispose maintenant de trois titres que certains d'entre vous ont peut-être eu l'occasion de voir à Expolangues et qui sont également en démonstration dans ces locaux : Sciences de la vie, Sciences de la matière, Agronomie et Agro-alimentaire.

\section{Les contraintes d'auteur}

Mais pour parvenir à ce produit dans son bel emballage il a fallu renoncer à plusieurs de nos ambitions ce qui a entraîné la démission d'un certain nombre d'auteurs qui considéraient les contraintes nouvelles comme autant d'atteintes à leur liberté créatrice : limites en nombre d'exercices, limites en quantité d'information apportée, limites en durée d'enregistrement sonore, mais aussi obligation systématique d'illustrations de type iconographique par exemple.

Comment, avec le recul, peut-on caractériser aujourd'hui les "contraintes » qui nous furent alors imposées? La contrainte, telle que nous la définissons maintenant, ce n'est que ce qui va à l'encontre de la finalité poursuivie par les auteurs. Loin de moi l'idée de comparer nos auteurs à Corneille ou Racine, mais il est sans doute bon de rappeler que la règle des 3 unités n'a pas, que je sache, empêché la création d'œuvres remarquables dans le cadre de la convention théâtrale. Est-ce donc une contrainte de dire à un auteur « Vous avez droit à $\mathrm{X}$ exercices de $\mathrm{N}$ caractères » ? Je répondrais qu'il s'agit plutôt du cadre du projet. Quitte à discuter cependant des limites du cadre chaque fois que cela est possible et apparaît nécessaire.

\section{Les contraintes d'utilisateur}

Dans EAO il y a A pour assisté. Pas plus que le polycopié ou la vidéocassette l'ordinateur et son logiciel ne sauraient remplacer l'enseignant. Par contre, plus que le polycopié, la vidéocassette ou tout autre support, l'ordinateur, de par les facilités d'hypernavigation qu'il propose et donc par sa convivialité apparente, impose une redéfinition de la fonction de l'enseignant traditionnel qui doit mettre en place une nouvelle relation d'apprentissage entre l'élève et lui face à la matière à acquérir.

Plus que détenteur et diffuseur de connaissances, mais ce n'est pas nouveau de le dire dans ce cadre où l'idée de Centres de ressource en autonomie guidée a déjà été discutée, l'enseignant doit devenir tour à tour professeur de méthodologie, conseiller et guide, souvent même auteur avec des exigences de perfection, je ne dirai pas de "contraintes", bien supérieures à celles d'un enseignement présentiel traditionnel.

31 Ce n'est pas nouveau de le dire, encore faudrait-il le faire. Sommes-nous tous prêts à assurer ce transfert de rôle? Le souhaitons-nous tous d'ailleurs?

\section{Conclusion}

Ma conclusion sera brève et reprendra en fait la question sous-entendue dès l'introduction. Vous avez certainement déjà entendu la boutade ayant accompagné la naissance du laser : une solution à la recherche d'un problème.

Quant à nous, les problèmes ne manquent pas, qu'il s'agisse de la matière ou des moyens de l'acquérir. 
il ne faudrait pas trop continuer le parallèle en considérant que les nouvelles technologies sont notre laser et qu'il suffit d'appliquer la machine au problème considéré, à savoir l'acquisition d'une langue, pour le résoudre. Même si le laser semble plus attirant, il faut savoir s'en servir et si, ce qu'à Dieu ne plaise, je devais demain subir une opération je préférerais un bistouri manié d'une main sûre à un laser mal utilisé.

\section{BIBLIOGRAPHIE}

Bacsich, P. et al. 1986. « Audio teleconferencing ». In Oxford Surveys in Information Technology, vol. 3. Oxford : Oxford University Press, 282-292.

Brodin, Elisabeth et Jean-Paul Narcy. 1991. «Comment choisir les nouvelles technologies en fonction des théories de la didactique des langues ». Actes $d u 12^{e}$ colloque $d u$ GERAS, Bordeaux : Université de Bordeaux, 11-15.

Carton, Marie-France. 1983. «Pour une didactique des stratégies conversationnelles ». Mélanges Pédagogiques, 53-102.

Derycke, Alain. 1991. «Réseaux et apprentissage coopératif : vers l'Université ouverte de $3^{\mathrm{e}}$ génération ». In La formation multimédia - Apprendre et former autrement. Actes du Colloque Synapse, 121-126.

Dudezert, Jean-Pierre. 1991. « Formation multimédia, coût du produit, coût du service ». In La formation multimédia - Apprendre et former autrement. Actes du Colloque Synapse, 249-253.

Garrison, D.R. 1990. « An analysis and evaluation of audio teleconferencing to facilitate education at a distance ». The American Journal of Distance Education 4/3, 13-24.

George, Judith. 1990. « Audioconferencing, Just another small group activity ». Educational and Training Technology International 27/3, 244-248.

Henri, France. 1988. « Formation à distance et communication assistée par ordinateur ». Perspectives 18/1, 87-93.

Kaye, Anthony. 1986. "La télématique comme outil de communication en formation à distance ». Le Bulletin de l'IDATE 23 mai.

Kaye, Anthony. 1992. «Collaborative learning through computer conferencing". In The Najaden Papers. Berlin : Springer-Verlag.

Kaye, Anthony et Claude Kerbrat. 1992. « Télécours d'anglais du tourisme ». Rapport d'évaluation . Montpellier.

Kirby, David et Cathryn Boak. 1989. «Investigating instructional approaches in audioteleconferencing classes ». Journal of Distance Education 4/1, 5-19.

Lagier, Laure. 1993. « Le cours à domicile par téléphone ». Rapport d'évaluation. Montpellier.

L'Henry-Evans, Odette. 1974. « Teaching by telephone: Some practical observations ». Teaching at a Distance 1, 67-69. 
Potter, Geoffrey. 1983. "The potential use of the telephone as instructional device in external studies ». Journal of Distance Education 4/1, 95-107.

Thibaut, Jean-Marie et Gérard Verrier. 1983. « Réalités et perspectives de l'utilisation de l'informatique dans l'enseignement des langues vivantes ». In Pratiques d'aujourd'hui et besoins de demain Actes du $4^{e}$ Symposium européen sur les langues de spécialité. Bordeaux : Université de Bordeaux, 109-118.

\section{ANNEXES}

\section{Annexe : Compte-rendu des discussions}

Annette Reumert-Kazes : Quel est le nombre d'auditeurs optimal pour un télécours, quelle est la durée d'un télécours, comment le suivi individuel est-il assuré, est-ce que tous les étudiants s'entendent les uns les autres?

Un groupe de 10 personnes autorise aussi bien un coût de fonctionnement acceptable qu'un apprentissage efficace car il y a presque toujours 1 ou 2 absents : nous nous adressons à des professionnels ayant des contraintes par ailleurs. Le télécours est prévu pour une durée d'une heure, mais il n'est pas rare que la séance dure $1 \mathrm{~h} 15$ car de nombreuses questions ou diversions nous éloignent parfois de l'objectif initial auquel nous tenons. Un cours plus long pourrait donc être envisagé, mais il faut tenir compte de la fatigue engendrée par l'attention soutenue. Quant au suivi, une liste des participants permet d'interroger systématiquement tout le monde à tour de rôle : le dialogue est géré par l'animateur

\section{Annie Bulko : Quel est le coût du système, qui paye les communications téléphoniques?}

Le coût dépend de l'emplacement du serveur. Chaque liaison (auditeur-serveur ou animateur-serveur) est basée sur les tarifs habituels de France Télécom. Au coût des communications, il faut ajouter la location et la maintenance du serveur, voire son amortissement, les frais de gestion, le salaire de l'enseignant, des frais divers de photocopie. On peut évaluer le coût à environ 100,00 F TTC de l'heure pour chaque participant, prix tout à fait compétitif pour un enseignement à distance interactif. C'est pourtant une somme trop élevée pour des individuels, il faut obligatoirement faire appel à des financements complémentaires extérieurs : département, région, Bruxelles par exemple.

\section{Janet Atlan : Quel genre d'exercices proposes-tu aux étudiants, y a-t-il un support} écrit ? Que fais-tu lorsque les participants ne comprennent pas un mot?

Il existe effectivement un polycopié : rappel grammatical élémentaire écrit par les auteurs et ce pour éviter des problèmes de copyright, exercices de grammaire avec corrigés. Mais le but est d'utiliser ces premiers documents en dehors des cours, en rappel de connaissances. Les participants ont évidemment toujours la possibilité d'interroger le formateur, mais le télécours ne doit pas se transformer en cours de grammaire. Un dernier document est la base du cours : listes de mots, images à décrire, exercices divers. Les exercices de compréhension orale sont soit pré-chargés sur le pont téléphonique soit simplement diffusés au dernier moment à partir du magnétophone de l'enseignant. Les exercices sont relativement traditionnels, mais découpés autrement en privilégiant les 
échanges rapides. Lorsqu'un mot n'est pas compris on l'épelle en anglais, on fait répéter, éventuellement en français, mais la pratique de l'alphabet est un des objectifs initiaux puisque nos participants doivent pouvoir prendre des messages par téléphone, noter des noms propres et pour cela l'alphabet est indispensable.

Elizabeth Jolivet : Le CD-ROM, à cause de son coût, répond-il vraiment à la situation concrète qui est la nôtre dans une université à savoir un grand nombre d'étudiants et un petit nombre de disques et d'ordinateurs?

Pour nous auteurs la situation est privilégiée : l'acquisition du CD-ROM se fait à prix coûtant d'une part et d'autre part le Ministère nous a alloué des crédits d'équipement nous permettant l'acquisition de 60 ordinateurs.

Par ailleurs le distributeur, Mediaconcept, s'adresse surtout à la Formation continue d'où les prix proposés. En tant qu'auteur et responsable pédagogique de l'ensemble du projet, je souhaite vivement, depuis 3 ans, que Mediaconcept développe une version réseau du produit ce qui par le biais des licences de site permettrait une utilisation au sein des Universités, IUT et autres établissements d'enseignement public. La technologie actuelle ne permet pas cette version pour l'instant.

Elizabeth Jolivet : Avez-vous des critiques à adresser à ce didacticiel ? Est-ce que le choix des modules dépend des professeurs de langues?

Oui, bien sûr. Regret d'abord de n'avoir pu développer tout de suite une version réseau. Regret aussi de ne pas proposer une plus grande variété d'exercices, mais le système est évolutif : tout ce qui est exercice peut être modifié, par les auteurs, si le besoin s'en fait sentir. Regret enfin de proposer toujours un même style de document : il s'agit de textes authentiques, mais alors que nous souhaitions proposer une typologie de textes, de situations d'énonciation différentes, nous avons été obligés pour des raisons pratiques de restreindre notre collaboration à un seul éditeur, Maxwell, d'où une certaine répétitivité. Mais cela ira mieux pour la suite !

Thomas Miller : Dans le cadre du télécours, y a-t-il plusieurs classes en même temps, est-ce que les étudiants sont choisis selon leur niveau et si oui comment, sont-ils classés selon leur profession, rédigez-vous des méthodes spécifiques?

Le pont téléphonique dispose d'un certain nombre de voies d'entrées qui définissent le nombre de classes possibles en parallèle. Pour un serveur à 16 voies comme celui de Mende on peut avoir une classe de 16, 2 classes de 8, etc.

Le recrutement se fait par le biais de la presse professionnelle puis la sélection s'opère à l'intérieur du public intéressé.

Quant aux méthodes, oui, nous écrivons ce dont les auditeurs ont besoin : nous avons eu une forte majorité d'hôteliers et restaurateurs, nous avons donc orienté notre production vers leurs besoins spécifiques. Nous préférons rédiger plutôt que de faire appel à des produits existants afin d'une part d'éviter les problèmes de copyright, d'autre part et surtout afin de viser au mieux à une adéquation des objectifs et des exercices d'apprentissage en fonction du temps dont nous disposons. 


\section{RÉSUMÉS}

Passé le premier émerveillement face aux capacités apparemment offertes par les outils technologiques, l'enseignant doit s'interroger sur leur emploi et leur valeur didactique. Deux exemples pris en langue de spécialité (anglais scientifique et anglais du tourisme) faisant appel à deux concepts (CD-ROM et téléphone) et à deux publics (formation initiale et continue) tenteront de montrer comment rechercher une adéquation entre les besoins, les moyens technologiques et l'approche pédagogique.

New technologies offer amazing possibilities. The teacher will not only wonder how to apply them but also, necessarily, analyze to what extent their use will be of didactic interest. Two examples chosen in ESP (science and tourism) requiring two media (CD-ROM and telephone) and aiming at two populations (initial and continuing education) will attempt to demonstrate how a concurrence should be reached between needs, technologies and pedagogical approach.

\section{INDEX}

Mots-clés : didacticiel, enseignement à distance, nouvelles technologies, télécours

Keywords : computer tutorial, new technologies, distance education, telecourse

\section{AUTEUR}

\section{JEAN-LOUIS TROUILLON}

Sciences et Techniques, Université Montpellier 2.jean-louis.trouillon@wanadoo.fr 